\title{
Palandt, Bürgerliches Gesetzbuch
}

\author{
Inhaltsübersicht
}
A. Ein neuer BGB-Kommentar
I. Das Konzept: Ein Kommentar im Stil der Baumbach-Reihe
II. Die Arbeit an der ersten Auflage des „Wilke“
III. Otto Palandt wird Herausgeber des Kommentars
IV. Otto Palandt, sein Lebensweg, seine Mitarbeit am Kommentar
V. Die ersten sechs Auflagen des Palandt
B. Der Neubeginn mit der 7. Auflage und die Fortsetzung des Erfolgs
I. Änderungen im Autorenteam
II. Die erste Nachkriegsauflage
III. Der Palandt, weiter der meist benutzte BGB-Kommentar
IV. Der Palandt und das Problem der „Einbändigkeit“
V. Der Palandt und die political correctness
C. Die midlife-crisis des Palandt und ihre Überwindung
I. Die midlife-crisis
II. Die Überwindung der Schwächeperiode
III. Weiterentwicklung von Auflage zu Auflage
D. Der Palandt im dritten Jahrtausend
I. Änderungen im Autorenteam
II. Das Schuldrechtsmodernisierungsgesetz
III. Schlußbemerkungen

\section{A. Ein neuer BGB-Kommentar}

\section{Das Konzept: Ein Kommentar im Stil der Baumbach-Reihe}

Die Geschichte des Buches, das den Namen „Palandt“ zu einem Warenzeichen und nach Änderung des Warenzeichenrechts zu einer Marke gemacht hat, begann vor mehr als 7 Jahrzehnten im Jahre 1934. ${ }^{1}$ In ihr spielte der Namensgeber, Otto Palandt, zunächst keine Rolle. 1933 hatte Heinrich Beck den Verlag Otto Liebmann in Berlin gekauft. ${ }^{2}$ Das wichtigste Verlagsobjekt, das Heinrich Beck übernommen hatte, war eine Reihe von systematisch aufgebauten, knapp und prägnant gefaßten Kommentaren. Wichtigster Autor, mit dem für die Reihe vorbildhaften Kom-

1 Der Verlag hat mir für meinen Beitrag eine Kopie der verlagsinternen Palandt-Chronik zur Verfügung gestellt, die Carl Hoeller, nach dem Zweiten Weltkrieg ,,Organisator und Zentralfigur des juristischen Lektorats“, verfaßt hat. Vgl. zu ihm Hans-Dieter Beck, Juristen im Portrait, 1988, S. 68. Außerdem habe ich die leider unvollständigen und lückenhaften Palandt-Archivalien des Verlages (4 Leitzordner) für die Zeit bis 1968 durchgesehen. Seit 1968 habe ich als PalandtAutor die Entwicklung des Kommentars miterlebt und mitgestaltet.

${ }^{2} \mathrm{Vgl}$. zu dieser einverständlich und fair zustande gekommenen Übernahme Heinrich Beck, Festschrift zum 200jährigen Bestehen des Verlages C. H. Beck, 1963, S. 123, $170 \mathrm{ff.}$ 
mentierungsstil war Adolf Baumbach, damals Senatspräsident am Kammergericht und Vorsitzender des Senats für Wettbewerbs- und Warenzeichensachen. Er hatte für den neuen Kommentartyp die Bezeichnung ,Kurzkommentar“ geprägt, ${ }^{3}$ eine Wortschöpfung, die Heinrich Beck 1935 für seinen Verlag beim Reichspatentamt als Warenzeichen eintragen und schützen ließ. Sieben der insgesamt dreizehn Kommentare dieser Reihe hatte Baumbach selbst verfaßt, vor allem die Kurzkommentare zur Zivilprozeßordnung, zum UWG und zum Handelsgesetzbuch. Seit 1924, dem Erscheinen des ZPO-Kommentars von Baumbach, war es ein fast schon typischer Ablauf: Wenn zu einem Gesetz ein Kurzkommentar neuer Konzeption erschien, drängte er in der Regel die bisher in der Praxis führenden Kommentare zurück und übernahm alsbald selbst die Führungsrolle.

Auch zum BGB hatte Otto Liebmann 1931 einen Kurzkommentar herausgebracht, den Otto Loening, James Basch und Ernst Straßmann geschrieben hatten. Er enthielt umfangreiche, aber wenig übersichtlich geordnete Rechtsprechungsnachweise. Der systematische Aufbau der Erläuterungen und ihre sprachliche Darstellung waren nicht immer gelungen, und es fehlte eine Herausarbeitung der leitenden Gesichtspunkte. Die Diskussion über eine Nachbesserung dieser Mängel wurde 1933 abrupt beendet. Der von drei jüdischen Juristen verfaßte Kommentar durfte nach der nationalsozialistischen Machtergreifung nicht in 2. Auflage herausgebracht werden.

Für den Verleger war nicht ernstlich zweifelhaft, was nun zu geschehen hatte. Die in der Kurzkommentar-Reihe beim BGB, dem größten, bedeutendsten und in der Praxis wichtigsten Gesetz, bestehende Lücke mußte geschlossen werden. Nach Beratungen mit Baumbach und gründlicher Abwägung des Für und Wider entschloß sich Heinrich Beck, in der Kurzkommentar-Reihe mit Berliner Autoren einen neuen Kommentar zum BGB herauszubringen. Er sollte ebenso wie die anderen Werke der Reihe systematisch aufgebaut, knapp und präzise gefaßt werden, gleichwohl aber die Grundgedanken der Rechtsinstitute herausarbeiten und die Judikatur zum BGB umfassend berücksichtigen und sorgfältig nachweisen.

\section{Die Arbeit an der ersten Auflage des "Wilke“}

Das Autorenteam des neuen Kurzkommentars wurde 1934 nach Vorschlägen von Baumbach zusammengestellt. Bei seiner Auswahl stellt Baumbach ausschließlich auf die fachliche juristische Qualifikation ab. Übergroße Nähe zum Nationalsozialismus war bei seinen Vorbehalten gegenüber dem Dritten Reich ${ }^{4}$ eher ein Argument gegen als für eine Berücksichtigung. Als Herausgeber und Gesamtredakteur wurde der Ministerialrat im Reichsjustizministerium Dr. Dr. Gustav Wilke gewonnen, ein hochbefähigter Jurist und früherer Richter am Oberlandesgericht Dresden. ${ }^{5}$ Die fünf

\footnotetext{
${ }^{3}$ Zu Baumbach vgl. Hefermehl, Juristen im Portrait, 1988, S. 130; zu Baumbachs Wortschöpfung „Kurzkommentar“, die 1931 die Bezeichnung Taschenkommentar ablöste, vgl. dort S. 131.

${ }^{4}$ Vgl. Hefermehl, Juristen im Portrait, 1988, S. 130, 136 und die dort referierte kritische Besprechung des sogenannten Dunlop-Urteils des Reichsgerichts durch Baumbach.

5 Vgl. zu ihm Gruchmann, Justiz im Dritten Reich, 1988, S. 245, 276. Er war Mitglied zahlreicher internationaler Gremien und Dozent an der Berliner Universität (DJ 1938, S. 799).
} 
Bücher des BGB wurden auf acht Autoren, überwiegend Kammergerichtsräte, verteilt, von denen zwei, Dr. Bernhard Danckelmann ${ }^{6}$ und Dr. Wolfgang Lauterbach ${ }^{7}$ für die weitere Entwicklung des Kommentars von besonderer Bedeutung werden sollten. ${ }^{8}$

Nach einer ersten Autorenbesprechung folgten vier Jahre harter Arbeit, zunächst des Stoffsammelns und Ordnens, dann des Konzipierens und Formulierens. ${ }^{9}$ Jeder Autor hatte etwa 250 Druckseiten zu schreiben, wobei es vor allem darum ging, auf möglichst wenig Platz möglichst viel Informationen unterzubringen und diese überzeugend systematisch zu gliedern. Einen zuarbeitenden Assistenten oder gar einen „Ghostwriter“ gab es nicht. Neben der Arbeit am Kommentar mußte ein volles, reichlich bemessenes Richterpensum bewältigt werden. Trotzdem war die Stimmung gut. Das Autorenteam hielt den Zeitplan und die Festlegungen über den Umfang der einzelnen Arbeitsabschnitte gewissenhaft ein. Der Herausgeber und Gesamtredakteur Wilke erwies sich als ein Glücksgriff. Er war ein perfekter Organisator und Koordinator und verstand es, alle während der Arbeit sichtbar werdenden Meinungsunterschiede auszugleichen. Die selbstbewußten Autoren hatten keinen Zweifel daran, daß ihr Erläuterungsbuch wesentlich besser werden würde als die damals gebräuchlichen Handkommentare von Achilles-Greif und Warneyer.

Lebhafte Diskussionen gab es darüber, welchen Namen der neue Kommentar erhalten sollte. Der Verlag und die Mehrheit der Autoren favorisierten zunächst den Titel „Kammergerichtsräte-Kommentar“. Sie gingen davon aus, daß die Rechtsprechung des traditionsreichen Kammergerichts kein geringeres Ansehen hatte als die des Reichsgerichts und waren der Auffassung, daß die Abnehmer und Benutzer den neuen Kommentar als eine - wegen der Verbindung von prägnanter Kürze und Vollständigkeit der Informationen - überlegene Alternative zum Reichsgerichtsräte-Kommentar ansehen würden. Es war vor allem Danckelmann, der diesen Überlegungen widersprach. Er hielt den Reichsgerichtsräte-Kommentar in der damals vorliegenden 8. Auflage für schwach und befürchtete, daß das negative Image des „RGRK“ auf den „KGRK“ abfärben könnte. Außerdem hatte er ein weiteres Argument, das sein gesundes Selbstbewußtsein verriet: Der Titel „Kammergerichtsräte-Kommentar“ sei auch deshalb fehl am Platz, weil die Mehrzahl der Autoren nicht lange Kammergerichtsräte bleiben, sondern in der juristischen Hierarchie weiter nach oben gelangen würden. Danckelmann setzte sich schließlich, von Wilke nachdrücklich unterstützt, durch. Als am 6. Dezember 1936 der schriftliche Verlagsvertrag abgeschlossen wurde, bestand allgemeines Einverständnis darüber, daß der neue Kommentar als der „Wilke“ herausgegeben werden sollte. Dem Buch den Namen des Herausgebers zu geben, entsprach auch der Tradition der Kurzkommentar-Reihe und bewahrte den Verlag bei der Auswahl von neuen Autoren vor unnötigen Beschränkungen, die sich aus der Verwendung des Titels „Kammergerichtsräte-Kommentar“ ergeben hätten.

\footnotetext{
${ }^{6} \mathrm{Zu}$ Danckelmann vgl. Heinrichs, Juristen im Portrait, 1988, S. 229.

7 Zu Lauterbach vgl. Albers, Juristen im Portrait, 1988, S. 511.

${ }^{8}$ Die weiteren Autoren waren Friesecke, Henke, Bunge, Seibert, Radtke und Pinzger.

${ }_{9}^{9}$ Danckelmann und Lauterbach haben mir in vielen Gesprächen den Ablauf der Arbeit an der ersten Auflage plastisch geschildert.
} 
Anfang 1938 war daher scheinbar alles klar. Alle Autoren hatten brauchbare Manuskripte beim Herausgeber abgeliefert. Alle gingen davon aus, daß die 1 . Auflage des „Wilke“, wie vereinbart, im Herbst 1938 ausgeliefert werden könne.

\section{Otto Palandt wird Herausgeber des Kommentars}

Aber es kam anders als von Verlag und Autoren geplant. Im Mai 1938 erlitt Wilke während einer Dienstreise in Österreich einen tödlichen Verkehrsunfall. Zu diesem Zeitpunkt lagen die Druckfahnen des neuen Kommentars praktisch vollständig vor. Die Autoren waren dabei, die Rechtsprechung und Literatur der letzten Monate in ihre Manuskripte nachzutragen. Durch den Tod Wilkes wurde der Verlag gezwungen, in kurzer Zeit einen neuen Herausgeber mit einem möglichst werbewirksamen Namen zu finden. Die Möglichkeit, einen der Autoren, etwa Danckelmann, Friesecke oder Lauterbach zum Herausgeber zu bestellen, wurde kurz erwogen, wegen des geringen Bekanntheitsgrades der Autoren aber verworfen. Eine solche Lösung hätte wohl auch Spannungen in das Autorenteam hineingetragen, da sich keiner der in Betracht kommenden Autoren durch Renommee, Rang oder Alter wesentlich von den anderen abhob.

Nach Beratung mit Baumbach trat Heinrich Beck daraufhin an Otto Palandt, den Präsidenten des Reichsjustizprüfungsamtes und Leiter der Abteilung Juristenausbildung des Reichsjustizministeriums heran und bot diesem an, die Funktion des Herausgebers des neuen Kommentars zu übernehmen. Palandt, bereits 61 Jahre alt und nicht ohne gesundheitliche Probleme, war sofort einverstanden. Nach dem Zusammenbruch des Nationalsozialismus hat die Entscheidung des Verlags, Palandt in einem Manöver des letzten Augenblicks zum Herausgeber zu bestellen, wiederholt zu Angriffen gegen Palandt, den nach ihm benannten Kommentar und den Verlag geführt. ${ }^{10}$ Der Leser sollte daher wissen, wer Otto Palandt war. In dem Beitrag über den Kommentar, der noch heute seinen Namen führt, darf somit ein Exkurs über Otto Palandt nicht fehlen.

\section{Otto Palandt, sein Lebensweg, ${ }^{11}$ seine Mitarbeit am Kommentar}

\section{Der Lebensweg}

Palandt wurde am 1. Mai 1877 in Stade als Sohn eines Taubstummenlehrers geboren. Seine Jugend verbrachte er in Hildesheim, damals Lebensmittelpunkt der Familie Palandt, dort ist eine Straße nach dem Großvater, dem Waisenhausinspektor der Stadt, benannt worden. Nach der an einem humanistischen Gymnasium bestandenen Reifeprüfung studierte Palandt an den Universitäten München, Leipzig und Göttingen Rechts- und Staatswissenschaften. Die Erste juristische Staatsprüfung bestand er 1899 und die Große juristische Staatsprüfung 1904, beide mit dem auch damals seltenen Prädikat ,gut“. Diese Examensergebnisse öffneten dem

\footnotetext{
10 Vgl. dazu Wrobel, KrJ 1968, S. 1 und Slapnicar, NJW 2000, S. 1692.

11 Vgl. Kulturwart, Beiträge zur deutsch-polnischen Nachbarschaft, Nr. 206, Febr. 1997, S. $33 \mathrm{ff}$.
} 
28jährigen 1905 den Weg in den preußischen Justizdienst. Er arbeitete als Richter am Amtsgericht Verden, am Amtsgericht Znin, damals ein Ort im preußischen Landkreis Bromberg, und am Landgericht Kassel. Im August 1915 nach Ausbruch des Ersten Weltkriegs begann ein Einsatz in der Provinz Posen und im besetzten Polen, seit 1916 im Rang eines OLG-Rats. Als der Einsatz 1919 endete, wurde Palandt das Amt eines OLG-Rats am Oberlandesgericht Kassel übertragen. Dort war er zeitweilig Beisitzer in einem Strafsenat, überwiegend war er aber in Zivilsachen tätig. Sein besonderes Interesse galt der Ausbildung des juristischen Nachwuchses. Als Mitglied des preußischen Justizprüfungsamtes gehörte er schon in den zwanziger Jahren des letzten Jahrhunderts zu den bekanntesten Prüfern in der Großen Juristischen Staatsprüfung.

Seine Dienstgeschäfte erledigte Palandt gut, zügig und zur Zufriedenheit seiner Dienstvorgesetzten. Gleichwohl, die bei überdurchschnittlich tüchtigen OLGRäten irgendwann fällige Beförderung zum Senatspräsidenten blieb aus. Als $\mathrm{Pa}$ landt am 1. Mai 1932 seinen 55. Geburtstag feierte, war er noch immer Oberlandesgerichtsrat. ${ }^{12}$ Seine letzte Beförderung lag 16 Jahre zurück. Aussichten, beruflich weiter voranzukommen, bestanden kaum noch.

Doch die nationalsozialistische Machtergreifung veränderte die Situation. Der neu ernannte preußische Justizminister Hanns Kerrl, früher ein Beamter des mittleren Justizdienstes, begann eine umfassende ,Säuberungsaktion“. ${ }^{13}$ Die durch die Entlassung und Umsetzung von jüdischen, sozialdemokratischen und demokratischen Juristen frei werdenden Stellen konnten nicht alle mit ,,alten Kämpfern“ besetzt werden, denn Bewerber, die bewährte Treue zum Nationalsozialismus mit dem auch nach Ansicht von Kerrl ${ }^{14}$ - erforderlichen Sachverstand verbanden, gab es nicht in großer Zahl. Begünstigt durch diese in grober Verletzung von rechtsstaatlichen Grundsätzen herbeigeführten Änderungen waren einige qualifizierte nationalkonservative Juristen. Für sie eröffneten sich unerwartet neue Aufstiegschancen.

$\mathrm{Zu}$ dem Kreis der durch diese Entwicklung Begünstigten gehörte auch Otto Palandt. Er wurde als Nachfolger von Schwister Präsident des preußischen Justizprüfungsamtes, das 1934 in das Reichsjustizprüfungsamt umgewandelt wurde. Wrobel und Slapnicar sind sich sicher, daß Palandt diese Beförderung Roland Freisler, damals Staatssekretär im preußischen Justizministerium, verdankt. ${ }^{15}$ Irgendeinen Beleg

\footnotetext{
12 Palandt war während seines Einsatzes im besetzten Polen mit wertlos gewordenem polnischem Ersatzgeld (polnischen Landesdarlehenskassenscheinen) besoldet worden. Er vertrat die Ansicht, daß das Deutsche Reich oder Preußen verpflichtet sei, die Darlehenskassenscheine einzulösen und hatte mit Klage gedroht. Slapnicar, NJW 2000, S. 1692, 1696 vermutet, daß diese Klagedrohung der Grund für die Nichtbeförderung Palandts war. Ich hoffe, daß diese für das preußische Justizministerium wenig schmeichelhafte Vermutung nicht zutrifft.

13 Gruchmann, Justiz im Dritten Reich, 1988, vgl. vor allem S. $221 \mathrm{ff}$.

${ }^{14}$ In der AV vom 15. 6. 1933 (JMBl S. 186) heißt es" In Fällen, in denen eine Beforderung eines führenden Mitglieds der NSDAP oder eine für diesen vorteilhafte Versetzung oder sonstige Vergünstigung in Frage kommt, ist besonders zu prüfen, ob diese Beförderung oder sonstige Vergünstigung nicht dem Grundsatz, daß der Kämpfer zuletzt an sich denkt, zuwiderläuft. Jedenfalls muß es vollkommen ausgeschlossen sein, daß lediglich die Zugehörigkeit zur NSDAP oder einer ihrer Formationen eine Beförderung oder sonstige Vergünstigung für den in Frage kommenden Beamten nach sich zieht.“

15 Wrobel, KrJ 1968, S. 1, 4 und Slapnicar, NJW 2000, S. 1692, 1696.
} 
dafür gibt es aber nicht. Zwar kannten sich der in Kassel als Rechtsanwalt tätige Freisler und Palandt. Möglicherweise war Freisler, vielleicht sogar während seiner bolschewistischen Phase, ${ }^{16}$ auch Referendar bei Palandt, aber auch das ist nicht belegt. Eine Freundschaft oder gar eine kumpelhafte Beziehung bestand zwischen beiden sicher nicht. ${ }^{17}$

Palandt trat 1933 der NSDAP bei und stellte sich nach außen ganz in den Dienst des nationalsozialistischen Staates. Zuvor war er ein gegenüber den Strukturen der Weimarer Republik kritischer Deutschnationaler. Antisemitismus war nicht seine Sache. Wie sich aus dem Bescheid des Hamburger Berufungsausschusses ,Justiz“ für die Ausschaltung von Nationalsozialisten vom 22. Dezember 1948 ergibt, hat Palandt im Rahmen seiner Möglichkeiten versucht, rassisch Verfolgten zu helfen, so Prof. Dr. Franz Leonhardt und dem Richterkollegen Konrad Hübner, der nach 1945 Landgerichtspräsident wurde. Natürlich gehörte Palandt als Präsident des Reichsjustizprüfungsamts zu den 850 Mitgliedern der Akademie für Deutsches Recht. ${ }^{18}$ Er hat aber offenbar in der Akademie und ihren 97 Ausschüssen nicht mitgearbeitet. Auch als am 30. Juli 1943 auf der Reichstagung der Akademie das Thema „Reform der juristischen Ausbildung“ behandelt wurde, trat Palandt nicht in Erscheinung. ${ }^{19} \mathrm{Im}$ übrigen gehörten zu den Mitgliedern der Akademie auch Goerdeler, Popitz und Jessen, die wegen ihres Widerstandes gegen den Nationalsozialismus ihr Leben lassen mußten.

Die von Palandt verfaßten Berichte über die Arbeit des Reichsjustizprüfungsamtes und die Prüfungsleistungen der Referendare enthielten immer ein heute unverständliches, aber damals wohl nicht zu vermeidendes Lob der nationalsozialistischen „Rechts“entwicklung. ${ }^{20}$ Schwerpunkt war jedoch immer eine substanzierte - manchmal ein wenig kleinkarierte - Kritik von Mängeln der Prüfungsleistungen, wie sie ähnlich auch heute abgegeben werden könnte.

Bei Kriegsende war Palandt 68 Jahre alt ${ }^{21}$. Er kam schon aus Altersgründen für eine Wiederverwendung im Justizdienst nicht in Betracht. Palandt, auch an philosophischen Fragen lebhaft interessiert, hatte schon in der nationalsozialistischen Zeit intensiv an einer „Klassischen Richtigkeitslehre“ gearbeitet. 1948 war sein umfangreiches Manuskript fertig. ${ }^{22}$ Es enthielt eine komplizierte Denktafel, die Grundlage der Metaphysik, Psychologie, Ontologie und Ethologie und der Gesamtwissenschaft werden sollte. Trotz heftigen Drängens des Autors war der Beck-Verlag aber nicht bereit, dieses inzwischen verschollene Manuskript zu veröffentlichen.

1948 wurde Palandt in der Britischen Zone entnazifiziert. Den Vorsitz im Berufungsausschuß führte der hochangesehene Hamburger Rechtsanwalt Dr. Emil

16 Vgl. zu ihr Gruchmann, Justiz im Dritten Reich, 1988, S. 64.

17 Das haben mir die Zeitzeugen Danckelmann, Lauterbach und Hoeller, damals als Gerichtsassesor und Landgerichtsrat im RJM tätig (s Gruchmann, Justiz im Dritten Reich, 1988, S. 81 Fn. 343), wiederholt bei Gesprächen berichtet.

18 Vgl. Pichinot, Die Akademie für Deutsches Recht, Kieler Dissertation, 1981.

19 Vgl. den Tagungsbericht in der ZAkDR 1943, S. 177.

20 Vgl. statt aller DJ 1937, S. 1645.

21 Die letzten Lebensjahre Otto Palandts werden in Durchbrechung des chronologischen Aufbaus des Berichts hier mitbehandelt.

22 Eine kurze Inhaltsangabe des Werkes befindet sich in den Beck-Archivalien. 
von Sauer. ${ }^{23}$ Der Ausschuß kam aufgrund der zahlreich vorgelegten Affidavits zu dem Ergebnis, Palandt sei in die Gruppe V „Entlastet“ einzustufen. Das Amt des Präsidenten des Justizprüfungsamtes sei ihm ausschließlich wegen seiner hervorragenden juristischen Qualifikation übertragen worden. Trotz des Gewichts, das die Mitwirkung Emil von Sauers dieser Entscheidung gibt, fällt es schwer, ihr zuzustimmen. Richtiger war wohl die Beurteilung der ersten Instanz, die Palandt in die große Gruppe der Mitläufer eingeordnet hat: Palandt hat sich zwar an nationalsozialistischen Unrechtshandlungen nicht beteiligt, er hat aber dem nationalsozialistischen Regime weiter gedient, obwohl ihm der fast völlige Abbau aller rechtsstaatlichen Grundsätze und die verbrecherischen Praktiken der neuen Machthaber nicht verborgen bleiben konnten.

Am 3. Dezember 1951 ist Palandt nach langer Krankheit im Alter von 74 Jahren gestorben. Die NJW widmete ihm einen kurzen Nachruf. ${ }^{24}$ Die übrigen juristischen Zeitschriften nahmen von seinem Tod keine Notiz.

\section{Mitarbeit am Kurzkommentar zum BGB}

Als Palandt die Funktion des Herausgebers übernahm, war die erste Auflage des Kommentars praktisch fertig. Sein einziger Beitrag zu dem nach ihm benannten Kommentar war die 18 Seiten lange Einführung. Sie bestand aus drei Teilen und war die wohl schwächste Partie des Werks. Im ersten, rechtsgeschichtlichen Teil legte Palandt dar, daß Sebastian Derrer, Vigilius von Aytta, Johann Oldendorp und Hermann Conring die bedeutendsten deutschen Juristen der Zeit vor Savigny waren. Das ist schwerlich richtig, gehört aber, selbst wenn es richtig wäre, nicht in die Einführung eines Kurzkommentars zum BGB. Der zweite Teil zählte alle Gesetzesänderungen seit dem Inkrafttreten des BGB auf. Der dritte Teil der Einleitung enthielt eine kritische Würdigung des BGB, die dem damaligen Zeitgeist entsprach und durch ihren geringen Tiefgang enttäuscht. Hoeller, ${ }^{25}$ ein Freund deutlicher Worte, hat später in einem Aktenvermerk des Verlags festgehalten, alle wüßten, daß Palandts Einführung ,miserabel“ sei. Eine Neufassung anzumahnen, bringe aber wahrscheinlich nichts, da sie möglicherweise noch schlechter werde. Vielleicht war Palandt schon 1939 so sehr mit seinem opus magnum „Klassische Richtigkeitslehre" ${ }^{26}$ beschäftigt, daß er sich für den Palandt kaum Zeit nahm.

Auch bei den weiteren Auflagen hielt sich Palandt bei der Arbeit erstaunlich zurück. Die Manuskripte der Autoren gingen nicht an den Herausgeber, sondern direkt an den Lektor des Verlags. Auftretende Probleme wurden von den Autoren in der Regel nicht mit dem Herausgeber, sondern mit dem Verlagsjuristen Flemming erörtert. Palandts Beitrag beschränkte sich auf minimale Änderungen der Ein-

${ }^{23} \mathrm{Zu}$ Emil von Sauer, Sohn des gleichnamigen Komponisten und Pianisten, vgl. Gerhard Commichau in „Recht und Juristen in Hamburg“, Bd. I, Teil V, 1997. Emil von Sauer, als sog. „Halbjude“ rassisch Verfolgter, war nach dem Krieg Neubegründer des Hamburgischen Anwaltvereins und des Deutschen Anwaltvereins. Der Hamburgische Anwaltverein ehrt Persönlichkeiten, die sich um das hamburgische und deutsche Rechtswesen verdient gemacht haben, durch Verleihung des nach ihrem Wiederbegründer benannten „Emil-von-Sauer-Preises“.

24 NJW 1951, S. 953.

25 Vgl. zu Hoeller, Fn. 1 und 17.

26 Vgl. zu ihr bei Fn. 22. 
leitung. Meistens ging es nur darum, die Aufzählung der Gesetzesänderungen ein wenig zu verlängern. Als Palandt, zuletzt auch durch Alter und Krankheit erheblich behindert, kurz vor Erscheinen der 10. Auflage starb, waren sich Verlag und Autoren rasch darüber einig, daß Palandts Position nicht wieder besetzt zu werden brauchte. Die Einführung ging in die Zuständigkeit des Autors für den Allgemeinen Teil über und wurde rasch deutlich besser. Die Aufgaben des Herausgeber wurden in unklarer, aber problemlos funktionierender Abgrenzung zwischen dem Verlag und der Autorenkonferenz geteilt, in letzterer wuchs dann irgendwann einem der Autoren die Stellung des Teamleaders zu.

\section{Die ersten sechs Auflagen des Palandt}

Anfang 1939 wurde die 1. Auflage des Palandt ausgeliefert. Der Verlag war sich des Erfolgs des Buches offenbar nicht recht sicher. Er ließ vom Palandt nur halb so viel Exemplare drucken wie für die erste, 1924 erschienene Auflage des ZPOKommentars von Baumbach. Das Vorwort, das natürlich auf Verbeugungen vor dem Nationalsozialismus nicht verzichten konnte, stellte schon im ersten Absatz klar, worum es im Kern ging. Der Palandt verstand sich als ein Kurzkommentar im Stil Baumbachs, er hatte die gleiche Konzeption wie der ZPO-Kommentar und wollte dessen Erfolg - 14 Auflagen in 14 Jahren - wenn möglich wiederholen. Er wandte sich nicht nur an Praktiker, sondern als Lehrkommentar auch an Studenten und Referendare.

Der Verkauf lief viel besser als vom Verlag erwartet. Die erste Auflage war in wenigen Tagen vergriffen. Im Vorwort der rasch erscheinenden 2. Auflage wurde daher von einem ,in der Geschichte des juristischen Verlagsbuchhandels einzig darstehenden Erfolg“ gesprochen. Auch in den folgenden Jahren blieb der Erfolg dem neuen BGB-Kommentar treu. Bis 1944 erschienen vom Palandt sechs Auflagen. Seit 1940 war der Palandt unter den Kommentaren zum BGB der Marktführer; er wurde am meisten benutzt und alsbald auch am meisten zitiert. $\mathrm{Zu}$ diesem Erfolg haben vor allem drei Gründe beigetragen:

\section{Schwächen der damals führenden Handkommentare}

Die in den dreißiger Jahren des letzten Jahrhunderts führenden Handkommentare zum BGB - der Achilles-Greiff und der Warneyer - hatten einen heute kaum noch verständlichen Kommentierungsstil. In beiden Kommentaren fehlte ein systematischer Aufbau. Im Achilles-Greiff wurden im Gesetzestext in der Regel an einige Worte hochgestellte Ziffern angehängt. Die Abfolge der Anmerkungen richtete sich nach diesen Ziffern und nicht nach Normzweck und Systematik der Vorschrift. Die Erläuterungen waren wenig transparent. In ihnen gab es kaum Absätze und Fettdruck. Noch trauriger sah es im Warneyer aus. Er bestand im wesentlichen aus einer nicht immer optimal geordneten Ansammlung von Leitsätzen der Rechtsprechung des Reichsgerichts. Beide Kommentare hatten den von Hermann Staub ${ }^{27}$

27 Vgl. zu seinem Kommentierungsstil Heinrichs, Deutsche Juristen jüdischer Herkunft, 1993, S. 385,393 . 
begründeten und von Adolf Baumbach für Kurzkommentare weiter entwickelten, heute völlig selbstverständlichen von allen Kommentatoren praktizierten modernen Kommentierungsstil noch nicht zur Kenntnis genommen.

\section{Die Leistung der Autoren}

Die Autoren des Palandt hatten gute Arbeit geleistet. Ihre Erläuterungen waren systematisch aufgebaut und übersichtlich und klar gegliedert. Die straffe Darstellung ermöglichte einen schnellen Zugang zu den Rechtsproblemen und den von Rechtsprechung und Lehre entwickelten Lösungsvorschlägen. In den Rezensionen der ersten Auflagen wurde lobend hervorgehoben, daß der Kommentar das systematische Element ganz in den Vordergrund rücke und ein Lehrkommentar im besten Sinne des Wortes sei. ${ }^{28}$ Professor Dr. Ernst Wolf hat später in einem Brief an den Verlag erklärt, daß er den Kommentar von Palandt für eine der bedeutendsten Leistungen der neueren Zivilrechtswissenschaft halte, der höchste Anerkennung gebühre.

\section{Der Name des Kommentars}

Der Name des Kommentars verlieh dem Werk bis zum Zusammenbruch des Dritten Reichs insbesondere bei Studenten und Referendaren erhebliche Zugkraft. Otto Palandt war damals als Präsident des Reichsjustizprüfungsamtes einer der bekanntesten deutschen Juristen und ein gefürchteter Prüfer. Da er häufig selbst den Vorsitz in der Großen juristischen Staatsprüfung übernahm, erschien es ratsam, den von ihm herausgegebenen Kommentar zur Examensvorbereitung zu benutzen. Die von Palandt im Vorwort des Kommentars gelobten Werke von Sebastian Derrer, Vigilius von Aytta, Johann Oldendorp und Hermann Conring sind wahrscheinlich nie wieder so häufig ausgeliehen und gelesen worden wie in den Jahren 1939-1945.

\section{B. Der Neubeginn mit der 7. Auflage und die Fortsetzung des Erfolgs}

\section{Anderungen im Autorenteam}

Als der Beck-Verlag 1947 nach dem Zusammenbruch des Dritten Reichs mit der Vorbereitung der 7. Auflage des Kommentars beginnen konnte, standen von den Autoren der ersten Stunde nur noch Danckelmann, inzwischen Rechtsanwalt in Frankfurt, und Lauterbach, nunmehr Richter am Hanseatischen Oberlandesgericht Hamburg zur Verfügung. Vier Palandt-Autoren - Friesecke, Henke, Radtke und Pinzger - waren im Kriege gefallen; zwei - Bunge und Seibert - waren an einer Mitarbeit nicht mehr interessiert. Als neue Autoren wurden Gramm, ${ }^{29}$ Senatspräsident in Hamburg, Hoche, Senatspräsident am Bayerischen Obersten Landesgericht und Rechenmacher, Ministerialrat im Bayerischen Justizministerium, gewonnen. In dem jetzt nur noch aus fünf Autoren bestehenden Team gab es mehr als ein Jahr-

28 Bach, JW 1939, S. 745.

29 Zu Gramm vgl. Heinrichs, Juristen im Portrait, 1988, S. 373. 
zehnt keine Änderungen. Danckelmann war für den Allgemeinen Teil und das Allgemeine Schuldrecht zuständig, Gramm für das Besondere Schuldrecht, Hoche für das Sachenrecht, Lauterbach für das Familienrecht und das Internationale Privatrecht und Rechenmacher für das Erbrecht.

\section{Die erste Nachkriegsauflage}

An der ersten Nachkriegsauflage war neben den Autoren und dem Verlag als strenge Zensurbehörde auch die ,publications branch“ der amerikanischen Militärbehörde beteiligt. Sie wachte mit Argusaugen darüber, daß kein nationalsozialistisches Wort, oder was man dafür hielt, publiziert wurde. Der Palandt wurde daher unter der energischen und zupackenden Leitung von Hoeller ${ }^{30}$ einer gründlichen Säuberung unterzogen. Alle nationalsozialistisch geprägten Vorschriften, Begriffe und Zitate wurden eleminiert. Auch politisch-staatsrechtliche Begriffe wie „Generalgouverment“ oder „Protektorat Böhmen und Mähren“ verschwanden, obwohl auf sie eigentlich, z.B. bei der Erörterung von Fragen der Staatsangehörigkeit, nicht verzichtet werden konnte. Erfreulicherweise stellte sich heraus, daß mehr als 90\% des Palandt-Textes auch bei Anlegung strengster Maßstäbe frei von nationalsozialistischen Begriffen und Gedankengut war.

Zugleich mit der Entnazifizierung des Textes war die Rechtsprechung und Literatur der ersten Nachkriegsjahre einzuarbeiten. Zu berücksichtigen war vor allem die Rechtsprechung des Obersten Gerichtshofs für die Britische Zone, aber auch die Aufsatzliteratur, die sich kritisch mit der Rechtsentwicklung der nationalsozialistischen Zeit auseinandersetzte. Im April 1949 war die 7. Auflage endlich fertig. Sie wurde ein Riesenerfolg. Rechtsanwälte und Richter brauchten auch nach 1945 ein im Stil Adolf Baumbachs verfaßtes Erläuterungsbuch zum BGB. Die Exemplare der 7. Auflage - der Verlag hatte dreimal soviel Exemplare gedruckt wie bei der ersten Auflage - waren rasch verkauft, und die Autoren begannen alsbald mit den Arbeiten an der nächsten Auflage.

\section{Der Palandt, weiter der meist benutzte BGB-Kommentar}

Ab 1949 brachte der Beck-Verlag vom Palandt jedes Jahr eine Neuauflage heraus. Der Palandt wurde zu einem von den Benutzern geschätzten Jahrbuch des Zivilrechts. Hauptaufgabe der Autoren blieb weiterhin, die immer umfangreicher werdende Rechtsprechung und Literatur in den Kommentar einzuarbeiten. Dabei stand seit 1950 natürlich die Rechtsprechung des BGH im Vordergrund. Die Autoren gingen, ohne daß darüber diskutiert wurde, von dem Grundsatz aus, daß der Palandt als inzwischen etablierter Standardkommentar zum BGB im Zweifel der BGH-Rechtsprechung zu folgen habe. Sie sahen es nicht als ihre Aufgabe an, besserwisserisch eine vom $B G H$ abweichende Linie zu verfolgen. Das bedeutete aber nicht, daß der Palandt dem BGH kritiklos folgte. Bedenken gegen die BGHRechtsprechung wurden zum Ausdruck gebracht. Ging es um praktisch oder

30 Vgl. zu Hoeller Fn. 1 und 17. 
dogmatisch wichtige Grundsatzfragen und war die Rechtsprechung des BGH mit der Rechtsüberzeugung des Autors schlechthin unvereinbar, wurde dem BGH nachdrücklich widersprochen. Das wichtigste, seit langem aber uninteressant gewordene Beispiel einer solchen Differenz war die Meinungsverschiedenheit zwischen dem IV. Zivilsenat des BGH und Lauterbach, damals einem der führenden Köpfe des Familienrechts, des IPR und des Zivilprozeßrechts, über die Auslegung des $§ 48$ Ehegesetz (Scheidung nach dreijähriger Heimtrennung). Sie hat der Gesetzgeber 1977 durch das Erste Gesetz zur Reform des Ehe- und Familienrechts im Ergebnis im Sinne Lauterbachs entschieden.

Neben der Rechtsprechung und Literatur hatten die Autoren fortlaufend Gesetzesänderungen und neue Gesetze zu berücksichtigen. In dem hier zu behandelnden Zeitraum bis 1965 sind vor allem das Wohnungseigentumsgesetz, das Gleichberechtigungsgesetz und das im Zuge des Abbaus der Wohnungszwangswirtschaft geänderte Mietrecht zu nennen. Es war fast selbstverständlich, daß alle zivilrechtlichen Neuerungen zunächst im Palandt kommentiert wurden. Die von den PalandtAutoren vertretenen Ansichten hatten in der Regel gute Aussicht, von der Rechtsprechung und der herrschenden Meinung übernommen zu werden, natürlich fast immer mit einer umfangreicheren und sorgfältigeren Begründung.

\section{Der Palandt und das Problem der „Einbändigkeit“}

Der intensive Anfall von Rechtsprechung und Literatur und die zunehmend zahlreicher werdenden zivilrechtlichen Gesetzgebungsakte machten es immer schwieriger, alle für die Arbeit des Rechtsanwalts und Richters notwendigen Informationen zum bürgerlichen Recht in einem Band unterzubringen. In Rezensionen und zahlreichen Zuschriften wurde dem Verlag nahegelegt, das Buch in zwei Bände zu teilen, einen ersten Band mit den drei ersten Büchern und einen zweiten Band mit den Büchern 4 und 5 und allen Nebengesetzen. Der Verlag und alle Autoren ${ }^{31}$ waren sich aber darüber einig, daß die Einbändigkeit des Werkes unbedingt erhalten werden müsse. Es gibt keinen ernsthaften Zweifel: Ein entscheidender Grund für den Erfolg des Palandt war und ist, daß er das ganze BGB und alle wichtigen Nebengesetze in einem Band übersichtlich kommentiert. Um mehr Informationen in einem Band unterzubringen, wurden die unterschiedlichsten Mittel eingesetzt. Die Verwendung von immer dünnerem Papier ermöglichte es, die Seitenzahl von ursprünglich gut 2000 auf 3000 zu erhöhen. Durch wiederholte Formatänderungen wurde erreicht, daß eine Palandt-Seite seit langem fast doppelt so viele Anschläge aufnehmen kann wie die Erstauflage. Hinzu kommen zwei weitere, häufig kritisierte, aber leider unvermeidliche Palandt-Besonderheiten. Die Verwendung von zahlreichen - entgegen den Behauptungen der Kritiker aber leicht verständlichen - Abkürzungen macht es möglich, im Palandt Informationen unterzubringen, für die sonst ein Zweitband von etwa 500 Seiten erforderlich wäre. Außerdem tragen die Palandt-Autoren durch eine unschöne, aber gut lesbare Stummelsprache dazu bei, daß der Palandt weiter einbändig bleiben kann.

31 Über eine Ausnahme ist später zu berichten; vgl. unten S. 398f. 


\section{Der Palandt und die political correctness}

In den sechziger Jahren des letzten Jahrhunderts begann eine lebhafte Diskussion über die Rolle der Juristen in der nationalsozialistischen Zeit und um die Frage, inwieweit die Justiz der Nachkriegszeit von ehemaligen Nationalsozialisten geprägt worden ist oder nicht. Am Rande dieser Diskussion ging es auch um den Palandt. ${ }^{32}$ Kritisiert wurde, daß der Kommentar, der sich in der Nazi-Zeit als Standardkommentar zum BGB durchgesetzt hatte, in der Bundesrepublik weiter der führende BGB-Kommentar geblieben war. Hinterfragt wurde auch die politische Belastung Palandts und der Palandt-Autoren und ihre Tätigkeit nach dem Zusammenbruch des Dritten Reichs. Das Interesse an diesem Thema tendiert inzwischen trotz eines Wiederbelebungsversuchs durch Slapnicar ${ }^{33}$ gegen Null. Trotzdem soll es hier nicht gänzlich ausgespart werden.

Wer sich in der nationalsozialistischen Zeit mit einer juristischen Problematik befaßte, konnte nur vom damaligen Gesetzesstand ausgehen. Wer das BGB kommentierte, mußte auch die Gesetze berücksichtigen, die Wieacker ${ }^{34}$ mit Recht als nationalsozialistische Terrorgesetze bezeichnet hat. Er konnte sich auch kaum der Tendenz widersetzen, durch „unbeschränkte Auslegung““35 von Generalklauseln und unbestimmten Rechtsbegriffen nationalsozialistisches Gedankengut in das Recht zu rezipieren. Alle Autoren, die in der Nazi-Zeit an juristischen Kommentaren zum BGB oder anderen Gesetzen gearbeitet haben, standen in diesen Zwängen und haben ihnen nachgegeben, auch die Autoren des Palandt. Der Palandt hat dem Nationalsozialismus aber keine stärkeren Konzessionen gemacht als die anderen damals erschienenen BGB-Kommentare. In den 16 Paragraphen des PalandtVerlagsvertrages vom 6. Dezember 1936 steht auch andeutungsweise nichts davon, daß das BGB im Sinne des Nationalsozialismus kommentiert werden sollte. Wie schon im ersten Absatz des Vorworts zur 1. Auflage klargestellt, ging es darum, zum BGB einen Kommentar des Baumbach-Typs auf den Markt zu bringen und an den Erfolg dieser Reihe, insbesondere des ZPO-Kommentars, anzuknüpfen.

Die Autoren des Palandt sind von dem unverdächtigen Adolf Baumbach ${ }^{36}$ ausgewählt worden. Es gibt keine Anhaltspunkte dafür, daß einer von ihnen an irgendeiner nationalsozialistischen Unrechtstat mitgewirkt hat. Sie mögen wie Otto Paland $t^{37}$ Mitläufer gewesen sein. Das interessiert aber heute nicht mehr. Vier Autoren der ersten Palandt-Generation sind im Zweiten Weltkrieg gefallen. Die vier Überlebenden haben nach 1945 am Wiederaufbau der Rechtspflege mitgearbeitet und sich vorbehaltlos und nachdrücklich zur Wertordnung des Grundgesetzes bekannt.

Entscheidend ist aber vor allem: Es ist unbestritten, daß unsere Privatrechtsordnung in den 60 seit 1948 erschienenen Palandt-Auflagen im Geist des Grundge-

\footnotetext{
32 Auch in Bremen war das Interesse groß. Ich habe zweimal mit Studenten der Universität Bremen über das Thema „Palandt“ diskutiert.

33 NJW 2000, S. 1692.

34 Wieacker, Privatrechtsgeschichte der Neuzeit, 1967, S. 533.

35 Rüthers, Die unbegrenzte Auslegung, 1968.

$36 \mathrm{Vgl}$. oben bei Fn. 4.

37 Vgl. oben bei Fn. 22.
} 
setzes kommentiert wird. Niemand hat seit 1948 im Palandt auch nur Spuren irgendeines nationalsozialistischen Gedankens entdeckt. Auch nur formell - durch eine Richtertätigkeit in der nationalsozialistischen Zeit - belastete Autoren gibt es seit einem Vierteljahrhundert beim Palandt nicht mehr. Das Thema Palandt und Nationalsozialismus sollte daher endgültig ad acta gelegt werden.

\section{Die midlife-crisis des Palandt und ihre Überwindung}

\section{Die midlife-crisis}

Der Erfolg des Palandt hielt in den sechziger Jahren des vorigen Jahrhunderts scheinbar ungebrochen an. Das Buch wurde zum Flaggschiff der Kurzkommentarreihe des Verlags. Die Rezensenten bezeichneten es als „Duden des Zivilrechts“, als „Jahrbuch des Rechts“, als „Meisterwerk der Kommentarkunst“ und als „Hauptstück unserer privatrechtlichen Literatur“, wobei natürlich der Wert derartiger Lobsprüche nicht allzu hoch angesetzt werden darf. Der 1952 erschienene BGB-Kommentar von Erman war und ist eine beeindruckende dogmatische Leistung, für den Palandt aber kein wirklicher Konkurrent. Die Auflagenhöhe des Palandt ging nicht zurück, sondern wuchs weiter.

Etwa Mitte der sechziger Jahre begann aber für den Palandt eine Phase, die man vielleicht als seine vorgezogene midlife-crisis bezeichnen kann. Für den kritischen Benutzer, wenn auch nur für ihn, wurden Defizite sichtbar. Sie betrafen allerdings nur die beiden ersten Bücher und das Familienrecht. Das Sachenrecht, für das Degenhart als Nachfolger Hoches zuständig geworden war, war vorzüglich, wenn auch für den Palandt zu umfangreich, kommentiert. Die Kommentierung des Erbrechts hatte Keidel, seit der 20. Auflage der Nachfolger Rechenmachers, im Ganzen erneuert und zu einem Glanzstück des Kommentars gemacht.

Dagegen boten die beiden ersten Bücher und das Familienrecht ein weniger positives Bild. Zwar war auch ihre Kommentierung weiter aktuell und zuverlässig. Alle Gesetzesänderungen wurden sorgfältig berücksichtigt, kaum eine wichtige Entscheidung übersehen. Die Übersichtlichkeit der Kommentierung begann aber zu leiden. Die 1934 konzipierte Gliederung stimmte in weiten Bereichen nicht mehr. Neuere Rechtsprechung und neueres Schrifttum wurde oft nur noch registriert, aber nicht mehr wirklich verarbeitet. Manche Anmerkungen verwandelten sich im Laufe der Jahre in eine schwer $z u$ durchschauende Aneinanderreihung von sich teilweise widersprechenden Rechtsprechungszitaten. Etwa 1966 oder 1967 gab es daher gute Gründe für die Befürchtung, daß der Palandt den Weg des AchillesGreiff und des Warneyer gehen könnte. Andere juristische Verlage standen in Wartestellung. Mindestens drei von ihnen bereiteten Konkurrenzerzeugnisse vor. ${ }^{38}$

38 Auch folgendes soll nicht unerwähnt bleiben: Der Verfasser war seit 1965 als Präsidialrichter für die Bücherei des OLG Bremen zuständig. Da ich als Mitglied des III. Senats des Gerichts fast ausschließlich Fälle aus dem Schuldrecht zu bearbeiten hatte und mir die Kommentierung des Schuldrechts im Palandt nicht mehr gefiel, ließ ich mich 1966 auf der Liste der Richter streichen, 


\section{Die Überwindung der Schwächeperiode}

Doch die Schwächeperiode des Palandt, von der Mehrzahl der Benutzer kaum bemerkt, ging rasch und folgenlos vorüber. Nach dem Tod Gramms übernahmen Thomas und Putzo in der 28. Auflage das Besondere Schuldrecht, beide damals Landgerichtsdirektoren in München und als Autoren eines neuen Erfolgskommentars zur ZPO bestens ausgewiesen. Der Verfasser, damals OLG-Rat in Bremen, wurde Nachfolger Danckelmanns. Das Allgemeine Schuldrecht ging in der 28. Auflage in meine Zuständigkeit über, die Kernmaterien des Allgemeinen Teils in der 30. Auflage. Nach dem Tod Lauterbachs traten in der 33. Auflage Diederichsen, Universitätsprofessor in Göttingen, und Heldrich, Universitätsprofessor in München in den Autorenkreis ein. Diederichsen übernahm das Familienrecht und Heldrich das Internationale Privatrecht.

Dem deutlich verjüngten Autorenteam gelang es in mehrjähriger intensiver Arbeit, die Alterserscheinungen des Kommentars abzubauen und die Kommentierung zu modernisieren. Durch umfassende Neubearbeitungen wurde der fachliche Standard des alten Palandt wieder erreicht, vielleicht durch einen verstärkten Dialog mit der Wissenschaft sogar übertroffen. Die angekündigten Konkurrenzerzeugnisse erschienen nicht; als dann mit erheblicher Verspätung doch zwei kurzgefaßte neue Erläuterungsbücher zum BGB herausgebracht wurden, war der Palandt bereits wieder so fest im Sattel, daß sein Erfolg nicht mehr nennenswert geschmälert werden konnte.

\section{Weiterentwicklung von Auflage zu Auflage}

Überschaut man die Weiterentwicklung des Kommentars bis zum Ende des zweiten Jahrtausends, ist nur noch Folgendes anzumerken:

\section{Weitere Änderungen im Autorenteam}

Da auch bei Palandt-Autoren die Leistungsfähigkeit irgendwann nachläßt, gab es im Autorenteam weitere Änderungen. Als Degenhart 1974 starb, wurde Bassenge, Vorsitzender Richter am Landgericht Lübeck, in der 35. Auflage sein Nachfolger. Durch den Tod Degenharts wurde ein seit Jahren schwelendes, nicht ganz einfaches Problem gelöst. Degenhart war ein hervorragender Jurist und Kommentator. Seine Darstellungskunst wird im Vorwort zur 34. Auflage mit Recht gelobt. Degenhart war aber nicht bereit, sich voll auf die Zwänge eines Kurzkommentars einzulassen. Seine Neubearbeitungen waren in der Regel mit Umfangvermehrungen verbunden. Den Kritikern hielt er entgegen, für ihn sei eine wissenschaftlich vollständige Kommentierung unverzichtbar, notfalls müsse der Palandt zweibändig erscheinen. Diese Diskussion brauchte nach Degenharts Tod nicht weitergeführt zu werden. Bassenge kürzte die Kommentierung des Sachenrechts und zeigte, daß das Niveau der Kommentierung dadurch nicht zu leiden brauchte.

die jährlich einen neuen Palandt erhielten. Für mich wurde eine neue Erman-Liste angelegt, die aber bald wieder geschlossen werden konnte, da ich als einziger Interessent 1967 nach Abschluß des Verlagsvertrags mit dem Verlag C. H. Beck reumütig zum Palandt zurückgekehrt bin. 
1981 begann Keidel, sich aus Altersgründen aus dem Palandt zurückzuziehen. Sein Nachfolger wurde Edenhofer, damals Richter am Oberlandesgericht München, der zunächst die Hälfte und ab der 43. Auflage das gesamte Erbrecht bearbeitete. Erfreulicherweise blieb das Erbrecht auch weiterhin ein Schmuckstück des Palandt.

Bis zur nächsten Änderung im Autorenteam vergingen 17 Jahre. Thomas, inzwischen Senior der Gruppe, entschloß sich 1998, seinen Arbeitsabschnitt in der Abfolge mehrerer Auflagen auf Sprau zu übertragen, damals Richter am Bayerischen Obersten Landesgericht, später Vizepräsident dieses hochangesehenen, inzwischen leider aufgehobenen Gerichts. Sprau arbeitet seit der 57. Auflage am Palandt mit und ist seit dem Tod Thomas Alleinautor der $\$ \S 631$ bis 852 . Er erneuerte zunächst die Kommentierung der Bürgschaft und anschließend die des Gesellschaftsrechts, Werkvertragsrechts und des Rechts der unerlaubten Handlung.

\section{Die familienrechtlichen Reformen}

Nach den ersten in Ruhe vorbereiteten familienrechtlichen Änderungsgesetzen dem Gleichberechtigungsgesetz und dem Nichtehelichengesetz - verfiel der Gesetzgeber bei der Reform des Familienrechts in eine hektische Betriebsamkeit. Durch das Adoptionsgesetz, das Erste Ehereformgesetz, das Sorgerechtsgesetz, das Unterhaltsänderungsgesetz, das Betreuungsgesetz, das Kindschaftsrechtsreformgesetz und zahlreiche weitere Änderungsgesetze wurde das Familienrecht im Ganzen neugestaltet. Vom ursprünglichen Normenbestand des vierten Buches blieben nur die Vorschriften unverändert, die die Vormundschaft über Minderjährige regeln. Diederichsen, seit 1974 im Palandt für das Familienrecht zuständig, hatte fortlaufend eine Vielzahl von neuen, dogmatisch und rechtstechnisch manchmal wenig gelungenen Vorschriften zu kommentieren. Eine weitere erhebliche Belastung ergab sich daraus, daß er zu jedem familienrechtlichen Änderungsgesetz für die NJW einen fundierten, von der Praxis hoch geschätzten Einführungsaufsatz verfaßte. Als Diederichsen 1998 sechs - zum Teil entgegen der ursprünglichen Planung vorzeitig verabschiedete - Änderungsgesetze zu kommentieren hatte, mußte er kapitulieren. Er teilte dem Verleger mit, daß er das Familienrecht erst Anfang 1999 abliefern könne; Diederichsen und Hans Dieter Beck verständigten sich darauf, daß die 58. Auflage ohne Familienrecht erscheinen und das Familienrecht im März des nächsten Jahres nachgeliefert werden sollte.

Als dieser für den Palandt wenig überzeugende, vielleicht sogar gefährliche Plan den übrigen Autoren mitgeteilt wurde, übernahm der Verfasser, inzwischen in der Gruppe der unbestrittene primus inter pares, das Krisenmanagement. Nach einigen Telefonaten stellten sich die Autoren der Bücher 1 bis 3 und 5 solidarisch als Nothelfer zur Verfügung und brachten die Kommentierung der $\S \S 1741$ bis 1921 durch überobligationsmäßige Anstrengungen auf den letzten Stand. Durch den gemeinsamen Einsatz aller Autoren, des Lektorats und der Druckerei wurde erreicht, daß die 58. Auflage entsprechend den üblichen Palandt-Abläufen Anfang Dezember 1998 mit einer vollständigen Kommentierung aller neuen Vorschriften ausgeliefert werden konnte. 
Zur Entlastung von Diederichsen wurde 1999 Brudermüller als Palandt-Autor gewonnen. Brudermüller, Richter am Oberlandesgericht Karlsruhe, durch zahlreiche familienrechtliche Veröffentlichungen ausgewiesen und inzwischen Präsident des Familiengerichtstags und Vorsitzender Richter am Oberlandesgericht, übernahm die Kommentierung des Abschnitts „Bürgerliche Ehe“ und überzeugte durch zahlreiche Neubearbeitungen.

\section{Randkorrekturen im Schuldrecht, Reform des Internationalen Privatrechts}

Während der Gesetzgeber die Rechtsfortbildung im Allgemeinen Teil und im Schuldrecht mehr als sieben Jahrzehnte lang im wesentlichen Rechtsprechung und Lehre überlassen hatte, wurden ab 1976 auch die ersten beiden Bücher Gegenstand des Reformprozesses. Dem 1976 verkündeten AGB-Gesetz folgte eine Reihe von weiteren, zur Umsetzung von EG-Richtlinien erlassenen Gesetzen: das Haustürwiderrufsgesetz, das Produkthaftungsgesetz, das Verbraucherkreditgesetz, das Teilzeit-Wohnrechtegesetz und das Fernabsatzgesetz. Bei der Kommentierung dieser Gesetze leisteten die Schuldrechtsautoren des Palandt Pionierarbeit. Sie waren in der Regel die ersten, die die neuen Vorschriften erläuterten. Das AGBGesetz wurde bereits in der im Dezember 1976 erschienenen 36. Auflage kommentiert, obwohl es erst am 1. April 1977 in Kraft trat.

Da Änderungen des Wirtschafts- und Schuldrechts in immer stärkerem Maße auf Rechtsetzungsinitiativen der EU zurückgingen, wurde die Entwicklung des Gemeinschaftsrechts von den Autoren sorgfältig beobachtet. Die Partien der Einleitung, die sich mit dem Gemeinschaftsrecht, seiner Bedeutung und seiner Auslegung befassen, wurden besonders gepflegt und waren stets auf der Höhe der Zeit.

Auch bei der Reform des Internationalen Privatrechts durch das IPR-Gesetz und das Gesetz zum IPR für außervertragliche Schuldverhältnisse war der Palandt das Buch der ersten Stunde. Heldrich legte als zuständiger Palandt-Autor in beeindruckend kurzer Zeit eine kompetente, prägnante und klar aufgebaute Kommentierung des neuen IPR vor. Es war ein weiteres Mal so, daß sich die Praxis auf den Palandt verlassen konnte und auch verlassen hat.

\section{Der Palandt im dritten Jahrtausend}

\section{I. Änderungen im Autorenteam}

$\mathrm{Zu}$ Beginn des dritten Jahrtausends brachte der Verlag die 60. Auflage des Palandt auf den Markt. Ihr folgten bis Ende 2006 sechs weitere Auflagen. Der lebens- und dienstälteste Autor Putzo begann in der 60. Auflage seinen Arbeitsabschnitt auf Weidenkaff, Vorsitzender Richter am Oberlandesgericht München, zu übertragen. Nach Erscheinen der 65. Auflage schied Putzo nach vier Jahrzehnten und 38. Auflagen endgültig aus dem Palandt-Team aus. Weidenkaff beeindruckte seine Mitautoren durch eine überzeugende Kommentierung des neuen Mietrechts und die zuverlässige Betreuung aller Partien seines schwierigen Arbeitsabschnitts. Der 
Arbeitsabschnitt von Heinrichs, jetzt Palandt-Senior, soll auf zwei Autoren übertragen werden. Grüneberg, seit 2006 Richter am Bundesgerichtshof, hat seit der 65. Auflage Teilpartien des Allgemeinen Schuldrechts übernommen und die Kommentierung mehrerer Vorschriften, z. B. die des $§ 311$, gründlich überarbeitet und verbessert. In der 68. Auflage wird sich Heinrichs voraussichtlich ganz aus dem Allgemeinen Schuldrecht zurückziehen, und bis dahin wird der Verlag auch einen neuen Autor für den Allgemeinen Teil gefunden haben. Durch diese beiden Wechsel vergrößert sich der Kreis der Autoren, die am Palandt in seiner mehr als siebzigjährigen Geschichte mitgearbeitet haben, auf 26.

\section{Das Schuldrechtsmodernisierungsgesetz}

Bei der Vorbereitung der 61. Auflage, die Ende 2001 erscheinen sollte, bestand für die Autoren und den Verlag eine nicht ganz einfache Situation. Das Bundesjustizministerium hatte den Entwurf des Schuldrechtsmodernisierungsgesetzes in das Gesetzgebungsverfahren eingebracht, das wichtigste und umfangreichste Änderungsgesetz zum BGB seit dem 1. Januar 1900. Während die Praxis (Bundesrechtsanwaltskammer, Deutscher Anwaltsverein, Richterbund, Instanzgerichte) dem Entwurf trotz Kritik in Einzelpunkten grundsätzlich zustimmte, wurde er von einem Teil der Hochschullehrer entschieden abgelehnt. Ob sich die Befürworter oder die Kritiker der Reform durchsetzen würden, erschien lange Zeit zweifelhaft. Nach einer heftigen kontroversen Diskussion verabschiedeten Bundestag und Bundesrat im Herbst 2001 das Gesetz. Es konnte am 29. November 2001 verkündet werden und am 1. Januar 2002 in Kraft treten.

Autoren und Verlag, die im Mai 2001 über Erscheinen und Inhalt der 61. Auflage entscheiden mußten, fanden für die Probleme des Palandt eine überzeugende und von den Abnehmern akzeptierte Lösung. Die 61. Auflage kommentierte auch im Allgemeinen Teil und im Schuldrecht das vor dem Inkrafttreten des Schuldrechtsmodernisierungsgesetzes geltende Recht. Dafür sprach auch, daß dieses Recht in Altfällen noch viele Jahre lang anzuwenden war. Das Gesetz zur Modernisierung des Schuldrechts wurde im Palandt-Stil - knapp, systematisch gegliedert, klar aufgebaut und mit vielen Nachweisen - in einem Ergänzungsband kommentiert, der im Frühsommer 2002 ausgeliefert wurde. Die Ende 2002 erschienene 62. Auflage kommentierte das ganze BGB mit Nebengesetzen und dem neuen Schuldrecht wieder in einem Band. Die von einigen Käufern geäußerte Befürchtung, der Ergänzungsband signalisiere, daß der Palandt nunmehr zweibändig werde, war unbegründet. Während sich die Mehrzahl der Sonderveröffentlichungen zur Schuldrechtsmodernisierung nur schleppend verkauften, brachten Rechtsanwälte und Richter der Kommentierung der Schuldrechtsreform im Palandt das in Jahrzehnten gewachsene Vertrauen entgegen. Von der 61. und 62. Auflage wurden mehr als je 70000 Exemplare verkauft, eine Auflagenhöhe, die sicher nicht wieder erreicht wird und die sich inzwischen auch wieder auf den früheren Stand von etwa 50000 Exemplaren ${ }^{39}$ zurückentwickelt hat. Die etwa 20 Sonderveröffent-

39 Vgl. dazu bereits Slapnicar, NJW 2000, S. 1692. 
lichungen zur Schuldrechtsreform hatten wahrscheinlich geringere Verkaufszahlen als die beiden einschlägigen Palandt-Auflagen.

\section{Schlußbemerkungen}

Wenn sich nach Putzo auch Heinrichs in den wohlverdienten Ruhestand zurückgezogen hat, wird das Palandt-Team aus neun Autoren bestehen, ein Autor für den Allgemeinen Teil, drei für das Schuldrecht, einer für das Sachenrecht, zwei für das Familienrecht, einer für das Erbrecht und einer für das Internationale Privatrecht. Die nach dem Ausscheiden der Senioren deutlich verjüngte Palandt-Mannschaft wird sich weiter an die in mehr als 70 Jahren bewährten Grundsätze halten, von denen mit unbedeutenden Modifikationen alle Palandt-Autoren ausgegangen sind. Sie sind conditio sine qua non für einen beständigen Erfolg des Buches:

1. Es versteht sich von selbst, die vereinbarten Ablieferungstermine werden auf den Tag genau eingehalten. Verzögerungen um Wochen, Monate oder gar Jahre, wie sie bei anderen Kommentaren immer wieder vorkommen, sind beim Palandt ausgeschlossen.

2. Der Autor muß durch eine umfassende Auswertung aller einschlägigen juristischen Periodika sicherstellen, daß neu anfallende Rechtsprechung und Literatur vollständig und an der systematisch richtigen Stelle in seiner Kommentierung eingearbeitet wird. Es muß weiter so bleiben, daß die Korrekturen, die die $\mathrm{Pa}-$ landt-Autoren im Jahresrhythmus vornehmen, in der Regel wirklich alle wichtigen Neuerungen abdecken.

3. Der Autor muß die Fertigkeit entwickeln, den Inhalt einer umfangreichen Entscheidung mit einem oft komplizierten Leitsatz kurz, möglichst in einem einzigen leicht verständlichen Satz, wiederzugeben. Er sollte sich dabei Zeit lassen und sich klar machen, daß die Formulierung, die sich zunächst aufdrängt, nicht immer die optimale ist.

4. Besondere Aufmerksamkeit muß er neuen Rechtsproblemen widmen, die die Praxis in einer Vielzahl von Fällen beschäftigen. Beispiele aus letzter Zeit sind etwa die Probleme des Vertriebs von „Schrottimmobilien“, Inanspruchnahme von Telekommunikationsdienstleistungen durch Angehörige, E-Bay-Verkäufe, neue Probleme der Schadensregulierung nach Verkehrsunfällen (Unfallersatztarif Berücksichtigung von Mehrwertsteuer im Fall fiktiver Schadensberechnung). Wenn die Problematik aus systematischen Gründen an verschiedenen Stellen zu behandeln ist, muß er dem Benutzer durch Verweisungen helfen.

5. Das Entstehen von neuen gesetzlichen Vorschriften muß der Autor von Anfang an sorgfältig beobachten. Wenn die Vorschrift - in der Regel nach einem mehrjährigen, mit einem Referenten- oder Diskussionsentwurf beginnenden Vorlauf - schließlich im Gesetzblatt erscheint, sollte die Kommentierung weitgehend fertig sein. Unverzichtbar ist dabei eine zuverlässige Hilfe bei der Materialbeschaffung. Sie wird seit mehr als 40 Jahren von der Lektorin des Palandt Gertrud Artmaier hervorragend sichergestellt.

6. Der Palandt kann seine Stellung als der am meisten benutzte und gekaufte BGB-Kommentar auf Dauer nur behaupten, wenn die Autoren Teile ihrer 
Kommentierung gelegentlich im Ganzen erneuern. Geschieht das nicht, besteht die Gefahr, daß aus den Erläuterungen durch das fortlaufende Einbessern von Rechtsprechungsnachweisen ein wenig übersichtlicher Entscheidungsbrei wird. Welche Teile jeweils erneuert werden, entscheidet der Autor. Er sollte es sich aber zur Regel machen, daß es eine Neuauflage ohne Neubearbeitungen nicht gibt.

7. Der Palandt ist nicht der einzige einbändige Kommentar zum BGB. Zu den konkurrierenden Werken kommen gelegentlich weitere hinzu. Konkurrenz ist ebenso wie Kritik für die Palandt-Autoren und den Verlag immer wieder Ansporn, den eigenen Kommentar zu überprüfen und $\mathrm{zu}$ verbessern. In den nächsten Auflagen stehen Autoren und Verlag vor der Aufgabe, die für den $\mathrm{Pa}-$ landt unverzichtbaren Kürzungen behutsam zu reduzieren und für sie ein stimmiges und transparentes Konzept zu entwickeln.

$\mathrm{Zu}$ überprüfen und innovativ weiterzuentwickeln ist auch der Internet-Auftritt des Palandt. Das inzwischen geschaffene digitale Palandt-Archiv könnte erweitert und durch Aufnahme zusätzlicher Informationen verbessert werden. Das Problem eines Palandt-Aktualitätendienstes schieben Verlag und die Autoren seit langem vor sich her, ohne daß sich bisher eine Lösung abzeichnet. Diese Frage darf im dritten Jahrtausend nicht ad calendas graecas vertagt werden. Es bleibt zu hoffen, daß sich das verjüngte Team bald mit dem Verlag auf eine überzeugende Lösung verständigt. Und natürlich hofft der Verfasser, daß die weiteren Palandt-Auflagen noch besser und noch erfolgreicher werden als die 39 Auflagen, an denen er 40 Jahre mitgearbeitet hat. 
https://doi.org/10.17104/9783406731181-385, am 26.04.2023, 09:28:49

Open Access - (oc) EY - http://www.beck-elibrary.de/agb 